\title{
De la politique, de l'économie et de la clinique au cas par cas entre partenaires!
}

\author{
R. Raggenbass, membre du Comité central de la FMH
}

1 J. Lacan cité par J.-A. Miller dans la revue «Cités: Philosophie, Politique, Histoire», ${ }^{\circ} 16,2003$, p. 111.

2 Dans cette idéologie de la maîtrise, même le «droit» au suicide risque d'être réglementé, supprimé (cf. le rapport d'avril $2005 \mathrm{du}$ Conseil fédéral sur le suicide et la prévention du suicide qui est une réponse au postulat Widmer).

3 Une langue toujours équivoque et source de malentendus. C'est cette prise en compte, par la clinique, du langage qui nous différencie le plus clairement de la médecine vétérinaire.

4 Le discours est insidieux parce qu'il fait du sujet un individu potentiellement coupable de ne pas être dans la norme du groupe. Il vise l'uniformisation et la suppression du singulier qui soit dit en passant fait la diversité du vivant.
«[...] la politique procède par identification, elle manipule des signifiants-maîtres, elle cherche par là à capturer le sujet. Celui-ci, il faut le dire, ne demande que ça [...].» [1]. Dans cette citation, l'équivoque porte évidemment sur le verbe manipuler.

A cette équivoque, les énoncés de la FMH n'échapperont pas. Cela dit, il est possible d'en tempérer les effets manipulatoires (au sens péjoratif cette fois) si les acteurs du champ de la politique de la santé s'engagent dans une réelle culture de partenariat qui aille au-delà des clichés qui circulent au sein de chaque camp et qui ont pour objectif de disqualifier les compétences et la capacité de compréhension des uns et des autres.

Actuellement, ce partenariat n'existe pas. Il n'est ni recherché, ni promu par le monde politique; celui-ci tente de s'orienter non pas à partir de sa responsabilité vis-à-vis de l'ensemble de la collectivité mais à partir des promesses de maîtrise prônées par les managers. Le moyen en est la manipulation de chiffres statistiques et de concepts; l'effet est de désigner l'autre comme responsable des échecs, ceci dans une logique de bouc émissaire. La FMH ne soutient évidemment pas cette orientation.

Pour tenter de sortir de son impasse, à savoir le manque de maîtrise, le politique a tourné son écoute de manière préférentielle et unidirectionnelle vers les managers qui, forts de leurs résultats économiques flamboyants (cf. Swissair, etc.) et leurs évidentes «capacités cliniques», nourrissent l'illusion certaine qu'une gestion strictement économique et concurrentielle du champ de la santé permettra de sortir de l'impasse et par là même redonnera à la politique la maîtrise de sa légitimité. Pour moi, il s'agit là d'une pure et simple démission de nos élus face à leurs responsabilités politiques.

La stratégie du management est donc prédominante dans le champ de la santé. Elle favorise l'illusion groupale de réussite et le discours du maître; elle a comme effet de rendre l'idée même du manque, de l'échec encore plus insupportable pour l'ensemble de la collectivité. Que vont faire les individus de leurs manques à être, dans une société où il devient de plus en plus difficile de s'insérer dans le monde professionnel avant 20 ans et après 45 ans? Où le beau, la forme physique, la bonne santé, la vie sans tabac et peutêtre bientôt sans alcool est réifié? Où ira se loger la tension liée à l'expression de la singularité lorsque les contrats d'assurance auront été truffés de clauses d'exclusion, que les soins auront été codifiés par pathologie et non par sujet, que l'AI et le chômage sauront enfin reconnaître à coup sûr le vrai du faux, ... Où ira se loger cette pression individuelle et finalement sociale lorsque dans cette orientation hygiéniste et sécuritaire tous ces champs du social auront été verrouillés par la folie objectivante d'un certain discours scientifique, politique et économique [2]? Comment vivrons-nous dans un monde dominé par l'idéologie de l'efficacité, de l'adéquation et de l'économicité accréditée? Dans cette dérive entrepreneuriale appliquée à la santé et au social, nos élus ont une responsabilité déterminante.

Il n'est pas question d'affirmer ici que la réalité du manager ne vaut rien. Il s'agit d'en pointer l'idéologie univoque, universalisante et déferlante appliquée à un domaine auquel il est inadapté; celui de la santé et du social. Inadaptée parce que cette idéologie univoque vise comme dans une entreprise de production classique - l'uniformité de l'objet de commerce, l'intégration de valeurs communes, l'identification à l'entreprise et par là même l'évacuation de la dimension conflictuelle propre au champ médico-social. Il nous appartient en effet de rappeler que la maladie n'est pas qu'un objet d'étude en soi, elle est aussi inscrite dans le corps d'une personne elle-même divisée par une langue [3]. Cela a nécessairement des effets de malentendus sur l'objectivité des soignants, des processus de soins et sur l'issue des traitements.

Il est intéressant de relever ici que la stratégie de la bonne pratique «managériale» prônée, soit celle issue de la «bonne fabrication» dans un contexte de libre concurrence entre objets comparables, ne se fonde plus aujourd'hui sur la domination autoritaire et la contrainte arbitraire d'une décision politique ou patronale mais sur un discours beaucoup plus subtil et insidieux [4] qui s'articule autour des signifiants d'autocontrôle, d'autocorrection et surtout d'auto-éva- 
luation. L'évaluation, dont l'apothéose est bien sûr l'accréditation, devient le maître mot de la politique appliquée à l'ensemble des champs sociaux. Je rends attentif que cette accréditation participe de la logique contemporaine du contrat qui tend à vider de son essence l'esprit de la Loi. «Dès qu'il y a contrat, il y a évaluation. Il y a évaluation a posteriori pour vérifier que le contrat a bien été respecté, mais il y a aussi évaluation a priori parce qu'il faut évaluer si le partenaire avec lequel on veut contracter est bien tel qu'on suppose qu'il est. C'est au fond ce moment-là qu'on désigne sous le nom d'accréditation.» [5] Cette évaluation se prétend aussi au service d'une démarche de qualité qui est fixée légalement (art. 32 de la LAMal) par le triptyque de l'économicité, de l'efficacité et de l'adéquation. Le tout est évidemment emballé et vendu dans un discours de responsabilisation que le politique confond encore souvent avec la bonne volonté [6]. L'histoire a déjà connu de tels signifiants-maîtres centrés sur l'auto-évaluation et les gouvernements qui le pratiquaient étaient tous situés dans les extrêmes du champ politique. Il est important que la FMH n'en soit pas dupe, qu'elle prenne en considération cet arrièreplan et qu'elle le dénonce.

Cette stratégie, qui se veut positive (logique du win-win), est un management de type participatif. Cela a pour conséquence que celui qui n’y participe pas est isolé, ségrégé, stigmatisé. Ce type de management est légitimé aujourd'hui non pas par un programme politique mais par le simple signifiant de la qualité qui en prend la place. Ce nom fétiche de «qualité» est maintenant sensé fédérer la collectivité et les professionnels de la santé. Le discours sur la qualité est cependant utilisé de manière perverse puisqu'il n'est plus possible d'interroger ce désir de qualité sans être suspecté d'hérésie contre le bien public? Le problème de cette position tient peut-être au fait que le moteur du discours sur la qualité n'est pas tant un défaut de qualité qu'il s'agirait urgemment de corriger (à ce jour personne ne peut démontrer que la médecine a pratiqué n'importe comment) mais bien le fantasme de la maîtrise des différences, des singularités et peut-être même des contestations sociales latentes qui, elles, coûtent très cher. Il est évident que si nous étions tous pareils et si nous nous comportions tous de manière standards, soit comme le discours du maître le voudrait, ce serait plus facile; vivement le clonage!

Dans cette nouvelle illusion groupale centrée autour du totem de la qualité et soutenue par un certain type de discours scientifique, il n'y a pas de place pour une culture de la gestion des différences fondée sur le fait clinique de la singularité ou du cas par cas. La communauté scientifique est désormais priée par l'économie (puisque le politique tend à démissionner) de fixer les critères économiques de compétence à partir de modèles éprouvés et testés dans l'industrie. Dans le capitalisme médical contemporain, le soignant n'est par conséquent plus encouragé à prendre la parole en son nom propre mais au nom de la norme élevée au rang de vérité universelle. Le patient aussi est prié d'offrir sa pathologie de manière standardisée. Si le soignant veut tout de même dire quelque chose, il faut qu'il soit prêt, pour être entendu, à s'engager dans de longues et toujours incertaines procédures judiciaires. Fort heureusement, un jugement très récent du Tribunal fédéral des assurances démontre que la justice est encore capable d'indépendance vis-à-vis de nos idéologues [7]!

Cela dit, le danger de cette standardisation ou uniformisation massive de ce qui est «la qualité» (et donc bien) pour la collectivité est la disparition du sujet, de la singularité de ses «imperfections» et du traitement au cas par cas au profit de la norme et de la statistique. Cette lente disparition se manifeste très clairement avec la diminution/limitation du temps de parole dans nos consultations; une réduction du champ de la parole et de l'écoute dont les médecines dites complémentaires ont très bien su faire leur niche. Cela se révèle également dans une certaine utilisation et lecture des guidelines issus d'une science basée sur l'évidence. Quelle évidence le singulier d'un sujet parlant peut-il donc bien avoir? Cette logique pousse les professionnels à inscrire leurs pratiques dans le cadre de véritables protocoles qui fixent leurs modes d'intervention. Cette protocolisation prétend renforcer les garanties d'une bonne pratique et donner une bonne conscience à tous les acteurs accrédités qui s'activent autour du franc dépensé dans la santé. Dans la recherche sur la qualité, la FMH et ses organisations de base ne sont pas inactives. Celles-ci cherchent à élaborer avec différents partenaires du champ de la santé de nouveaux concepts - incluant le sujet - pour définir et gérer la qualité en médecine [8]. Ce partenariat est cependant encore très balbutiant et fragile en raison d'a priori tenaces et du manque de soutien politique. Mais dans ce travail, aucune question, aucun thème n'est en soi tabou pour la FMH.

Pour conclure, il me semble que la réflexion sur la qualité ne peut être conduite que si la dimension subjective y est intégrée et que la recherche articule tous les partenaires impliqués. Cette articulation ne peut s'exercer que si chaque acteur peut se faire entendre comme un partenaire responsable de ses dires et des consé- 
9 Corps médical définis dans la Loi sur les professions médicales. Quant au sens de «en tous les cas»; il signifie que cette énumération n'est pas exhaustive. quences de ceux-ci. Ce n'est qu'à cette condition que ceux-ci pourront être reconnus comme des partenaires dans l'ensemble des négociations portant sur le champ du social et de la santé. Dans ce sens, la FMH demande à ce que soit réellement mis en place et promu, à partir du Conseil fédéral et au niveau du Parlement, une culture de partenariat dans laquelle chaque acteur puisse loger son dire et ses compétences. Le partenariat souhaité concerne en tous les cas les politiques, les assureurs, le corps médical [9] et les usagers. Il implique que chaque camp sorte de son discours de maître qui, par définition est non partenarial, puisqu'il ne peut être qu'unique. Sortir de cette position ne peut avoir lieu que si l'on accepte de perdre quelque chose au passage, quelque chose du registre de l'illusion de la maîtrise (évoqué plus haut) et non quelque chose du réel de la responsabilité politique qui doit demeurer chez nos élus et en fin de compte chez le citoyen.

\title{
Von Politik, Wirtschaft und klinischer Einzelfallbetrachtung unter Partnern!
}

\author{
R. Raggenbass, Mitglied des Zentralvorstands
}

$1 \ll[\ldots]$ die Politik identifiziert und manipuliert Herrensignifikanten und versucht dadurch das Subjekt $\mathrm{zu}$ fassen. Allerdings verlangt das Subjekt auch genau das [...].» (Übersetzung FMH) J. Lacan zitiert von J.-A. Miller in der Zeitschrift «Cités: Philosophie, Politique, Histoire», Nr. 16, 2003, S. 111.
«[...] la politique procède par identification, elle manipule des signifiants-maîtres, elle cherche par là à capturer le sujet. Celui-ci, il faut le dire, ne demande que ça [...]». [1] Die Zweideutigkeit in diesem Zitat liegt natürlich beim Verb «manipulieren».

Dieser Zweideutigkeit entgehen auch die Äusserungen der FMH nicht. Die manipulative Wirkung im negativen Sinne kann freilich gemildert werden, wenn die gesundheitspolitischen Akteure eine echte Partnerschaftskultur aufbauen, welche die Klischees jeden Lagers überwindet, mit denen versucht wird, die Kompetenzen und Verständnisfähigkeit des anderen in Misskredit zu bringen.

Zurzeit gibt es keine solche Partnerschaft. Sie wird von der Politik weder angestrebt noch gefördert. Diese versucht sich nicht an ihrer Verantwortung gegenüber der Allgemeinheit zu orientieren, sondern an den Versprechungen der Manager, dass sie die Lage beherrschen. Dazu manipulieren sie statistische Angaben und Konzepte. Das hat zur Folge, dass der andere für das Versagen verantwortlich gemacht und zum Sündenbock abgestempelt wird. Die FMH unterstützt natürlich eine solche Ausrichtung nicht.

Um aus der Sackgasse, d. h. der mangelhaften Beherrschung, herauszukommen, wendet sich die Politik vorzugsweise und einseitig an die Manager, die, gestützt auf ihre glänzenden wirtschaftlichen Ergebnisse (vgl. Swissair usw.) und ihre offensichtlichen «klinischen Fähigkeiten», die Illusion hegen, dass eine streng wirtschaftliche und wettbewerbsorientierte Verwaltung des Gesundheitswesens einen Weg aus der verfahrenen Situation darstellt und dadurch der Politik wieder zu legitimer Steuerungshoheit verhilft. Für mich handelt es sich dabei schlicht und einfach um eine Demission unserer Volksvertreter vor ihrer politischen Verantwortung.

Im Gesundheitswesen herrscht also die Managementstrategie vor. Sie begünstigt eine kollektive Erfolgsillusion sowie eine Machtpositionierung und hat zur Folge, dass schon die Vorstellung einer Unzulänglichkeit oder eines Versagens für die Allgemeinheit absolut unerträglich wird. Was werden die Menschen mit den Unzulänglichkeiten ihres Lebens in einer Gesellschaft machen, in der die Eingliederung in die Berufswelt im Alter von unter 20 und über 45 Jahren immer schwieriger wird? In einer Welt, in der Schönheit, körperliche Verfassung und gute Gesundheit sowie ein rauchfreies und vielleicht bald auch alkoholfreies Leben verherrlicht werden? Wo wird die Spannung, die mit dem Ausdruck der Einzigartigkeit verbunden ist, noch Platz finden, wenn die Versicherungsverträge vor Ausschlussbestimmungen strotzen, die Pflege nach Pathologie statt nach Patient kodiert wird und IV und Arbeitslosenversicherung zu 100\% die «Echten» von den «Falschen» unterscheiden zu können glauben? Wo kann dann der indivi- 
duelle und letztendlich kollektive Druck noch Platz finden, wenn bei dieser Ausrichtung auf Hygiene und Sicherheit alle Sozialbereiche durch den Objektivierungswahn eines bestimmten wissenschaftlichen, politischen und wirtschaftlichen Diskurses abgeschottet werden? [2] Wie werden wir in einer von der Ideologie der akkreditierten Wirtschaftlichkeit, Wirksamkeit und Zweckmässigkeit beherrschten Welt leben? Für diesen unternehmerischen Holzweg im Gesundheits- und Sozialbereich sind unsere Volksvertreter entscheidend mitverantwortlich.

Es geht hier nicht darum, zu behaupten, dass die Sichtweise des Managers nichts wert ist, sondern darum, auf deren eindeutig, verallgemeinernd und überschwappend eingesetzte Ideologie hinzuweisen, die auf ein Gebiet (Gesundheits- und Sozialwesen) angewandt wird, für das sie nicht geeignet ist. Sie ist ungeeignet, weil sie wie in einem klassischen Produktionsbetrieb die Einheitlichkeit des Handelsguts, die Integration gemeinsamer Werte, die Identifizierung mit dem Unternehmen und dadurch die Beseitigung der Konfliktdimension des medizinisch-sozialen Bereichs anstrebt. Wir müssen daran erinnern, dass eine Krankheit nicht nur ein Studienobjekt ist, sondern auch im Leib einer Person steckt, die wiederum durch die Sprache gespalten erscheint [3]. Dies wirkt sich missverständlich auf die Objektivität von Pflegenden und Pflegeprozessen sowie auf den Ausgang von Behandlungen aus.

Interessanterweise stützt sich die befürwortete Strategie der guten Managerpraxis, die aus dem Konzept der «good manufacturing practices» in einem Umfeld des freien Wettbewerbs zwischen vergleichbaren Gütern stammt, heute nicht mehr auf die autoritäre Vorherrschaft und den willkürlichen Zwang einer Entscheidung der Politik oder der Arbeitgeber, sondern auf einen viel subtileren und heimtückischeren [4] Diskurs rund um die Kriterien Selbstkontrolle, Selbstkorrektur und vor allem Selbstevaluierung. Die Evaluierung, deren Krönung natürlich die Akkreditierung darstellt, wird zum Zauberwort der in allen Bereichen des Sozialwesens verfolgten Politik. Es sei darauf hingewiesen, dass diese Akkreditierung von der heute herrschenden Logik des Vertrags ausgeht, die dazu neigt, den Geist des Gesetzes seiner Substanz zu berauben. «Sobald ein Vertrag vorhanden ist, gibt es auch eine Evaluierung, und zwar eine Evaluierung im Nachhinein, um zu überprüfen, ob der Vertrag eingehalten worden ist, aber auch eine Evaluierung im Vorfeld, weil man abschätzen muss, ob der Partner, mit dem man einen Vertrag abschliessen will, auch wirklich so ist, wie man vermutet. Im
Grunde genommen ist das der Augenblick, der als Akkreditierung bezeichnet wird.» [5] Die Evaluierung gibt auch vor, der Qualitätssicherung zu dienen, die mit dem Dreigestirn Wirtschaftlichkeit, Wirksamkeit und Zweckmässigkeit gesetzlich vorgeschrieben ist (Art. $32 \mathrm{KVG}$ ). All dies wird natürlich in einen Diskurs über die Schaffung eines von der Politik noch oft mit gutem Willen verwechselten Verantwortungsbewusstseins verpackt und verkauft [6]. Solche auf die Selbstevaluierung ausgerichteten Hauptmerkmale hat es bereits früher gegeben, und alle Regierungen, die sich darauf gestützt haben, waren am äussersten Rand des politischen Spektrums angesiedelt. Die FMH darf sich nicht in die Irre führen lassen, muss diesen Hintergrund berücksichtigen und anprangern.

Diese Strategie, die sich positiv versteht (Win-Win-Logik), ist eine Form des partizipativen Managements. Die Folge ist, dass diejenigen, die nicht mitmachen, isoliert, ausgegrenzt und stigmatisiert werden. Diese Managementform wird heute nicht durch ein politisches Programm, sondern stattdessen einfach durch das Kennzeichen Qualität legitimiert. Dieses Fetischwort «Qualität» soll nun die Allgemeinheit und die Gesundheitsfachleute zusammenführen. Der Qualitätsdiskurs wird jedoch auf pervertierte Weise geführt, weil dieses Streben nach Qualität nicht mehr in Frage gestellt werden kann, ohne des Verrats am Gemeingut verdächtigt zu werden. Das Problem bei dieser Haltung liegt vielleicht darin, dass die treibende Kraft hinter diesem Diskurs nicht so sehr ein dringend zu behebender Qualitätsmangel ist (bisher konnte jedenfalls niemand beweisen, dass in der Medizin einfach irgendwie praktiziert worden ist), sondern das Trugbild der Beherrschung der Unterschiede, der Einzigartigkeit und vielleicht sogar der latenten sozialen Proteste, die uns sehr teuer zu stehen kommen. Eines ist klar: Wenn wir alle gleich wären und uns standardmässig verhielten, d.h., so wie der «Herr» es will, wäre es einfacher: Das Klonen lässt grüssen!

In dieser neuen Kollektivillusion rund um das Totem der Qualität, die von einer bestimmten Art des wissenschaftlichen Diskurses unterstützt wird, ist kein Platz für eine Kultur, die mit Unterschieden auf der Basis des klinischen Einzelfalls oder der Einzigartigkeit umzugehen weiss. Die Wissenschaft wird nun (weil die Politik sich ihrer Verantwortung eher entzieht) von der Wirtschaft aufgefordert, die wirtschaftlichen Kompetenzkriterien auf Grund von in der Industrie bewährten und getesteten Modellen festzulegen. Im heutigen medizinischen Kapitalismus wird der Arzt folglich nicht mehr dazu er- 
7 Vgl. den kürzlich getroffenen Entscheid des Eidgenössischen Versicherungsgerichts, das anerkannte, dass die Grundversicherung auch die ambulante Krankenpflege in der Psychiatrie übernehmen muss, was die Versicherer abgelehnt hatten.

8 Peltenburg M, Kernen $\mathrm{H}$, Schneider P, von Below G, Waldis G, Vogel HA, et al. Qualität: ein Zusammenspiel aller Kräfte im ärztlichen Umfeld. Schweiz Ärztezeitung 2005;86(18):1055-8. Man kann nur hoffen, dass unsere Kollegen die obenerwähnten Klippen der Beherrschung zu umschiffen wissen.

9 Ärzteschaft gemäss Definition im Bundesgesetz über die universitären Medizinalberufe. «Auf jeden Fall» bedeutet, dass diese Aufzählung nicht abschliessend ist. mutigt, in seinem eigenen Namen, sondern im Namen der zur Universalwahrheit erhobenen Norm zu sprechen. Auch der Patient wird gebeten, seine Krankheit gefälligst standardisiert anzubieten. Wenn der Arzt trotzdem etwas sagen will, muss er, um sich Gehör zu verschaffen, bereit sein, lange gerichtliche Verfahren mit ungewissem Ausgang in Kauf zu nehmen. Glücklicherweise zeigt ein kürzlich getroffener Entscheid des Eidgenössischen Versicherungsgerichts, dass die Justiz noch zur Unabhängigkeit von unseren Ideologen fähig ist! [7]

Die Gefahr der massiven Standardisierung und Vereinheitlichung dessen, was für die Allgemeinheit die «Qualität» (und somit etwas Gutes) darstellt, ist das Verschwinden des Subjekts, der Einzigartigkeit seiner «Unzulänglichkeiten» und der fallweisen Behandlung zugunsten von Norm und Statistik. Dieses schleichende Verschwinden zeigt sich klar darin, dass bei unseren Konsultationen die Zeit für das Gespräch immer stärker zu kurz kommt. Den abnehmenden Raum für Gespräch und Zuhören hat die sogenannte Komplementärmedizin denn auch für sich als Nische entdeckt und zu nutzen verstanden. Es zeigt sich auch in einer gewissen Art der Verwendung der aus der evidenzbasierten Wissenschaft stammenden Richtlinien. Was für eine Evidenz kann das Einzigartige eines sprechenden Subjekts haben? Diese Logik bringt die Fachleute dazu, ihre Praxis in regelrechte Protokolle zu zwängen, die ihre Interventionen bestimmen. Diese «Verprotokollierung» gibt vor, für eine gute Qualität $\mathrm{zu}$ sorgen, und verhilft allen akkreditierten Akteuren, die sich um jeden im Gesundheitswesen ausgegebenen Franken kümmern, zu einem guten Gewissen. Beim Streben nach Qualität sind die FMH und ihre Basisorganisationen nicht untätig geblieben. Sie versuchen, mit verschiedenen Partnern des Gesundheitswesens neue Konzepte auszuarbeiten, die das Subjekt einbeziehen, um die Qualität in der Medizin zu definieren und zu sichern [8]. Diese Partnerschaft steckt aber erst in den Anfängen und ist wegen hartnäckiger Vorurteile und mangelnder politischer Unterstützung noch zerbrechlich. Bei dieser Arbeit ist jedoch für die FMH keine Frage, kein Thema tabu.

Abschliessend scheint mir, dass Überlegungen über die Qualität nur angestellt werden können, wenn die subjektive Dimension berücksichtigt wird und die Forschung alle betroffenen Partner beteiligt. Dies kann jedoch nur erfolgen, wenn jeder Akteur sich als Partner Gehör verschaffen kann und Verantwortung für seine Worte und deren Folgen übernimmt. Einzig unter dieser Voraussetzung können sie als Partner bei allen Verhandlungen im Sozial- und Gesundheitswesen anerkannt werden. In diesem Sinne fordert die FMH, dass ausgehend vom Bundesrat und Parlament eine echte Partnerschaftskultur aufgebaut wird, in der alle Betroffenen etwas zu sagen haben und ihre Kompetenzen einbringen können. Die gewünschte Partnerschaft betrifft auf jeden Fall die Politiker, Versicherer, Ärzteschaft [9] und Patienten. Dies setzt voraus, dass jedes Lager von seinen bisher sakrosankten - zwangsläufig nicht partnerschaftlichen, sondern einseitigen - Zielsetzungen abrücken kann. Diese Haltung kann aber nur derjenige gewinnen, der bereit ist, etwas aufzugeben, etwas im Bereich der obenerwähnten Beherrschungsillusion und nicht etwas Reales auf dem Gebiet der politischen Verantwortung. Diese muss bei unseren Volksvertretern und letztendlich beim Bürger bleiben. 